\title{
Política exterior y derechos humanos en México: tendencias a finales del siglo XX y prioridades a inicios del $\mathrm{XXI}^{*}$
}

\section{Rafael Velázquez Flores**}

\section{RESUMEN}

El objetivo central de este artículo es identificar las causas que llevaron a México a incluir el tema de los derechos humanos como un punto destacado en su agenda de política exterior. El argumento principal es que esa decisión se debió a la mezcla de factores tanto externos como internos que tuvieron lugar a finales del siglo xx. El trabajo está dividido en cuatro partes. La primera plantea el vínculo entre politica exterior y derechos humanos desde una perspectiva conceptual. La segunda explica los factores externos que motivaron a México a proyectar una política exterior distinta en materia de derechos humanos a finales del siglo xx. La tercera analiza los elementos internos que incidieron. La última parte analiza algunos casos especificos sobre la vinculación entre política externa y derechos humanos en el caso mexicano.

PALABRAS CLAVE: Derechos humanos, política exterior, México, sistema internacional, democracia.

\section{ABSTRACT}

The central target of this article is to identify the causes that led Mexico to including the topic of human rights as a significant point in its foreign policy agenda. The main argument is that this decision was due to the mix of external and internal factors that took place at the end of the twentieth century. The work is divided into four parts. The first one raises the link between foreign policy and human rights from a conceptual perspective. The second one explains external factors that motivated Mexico to project a different foreign policy on the field of human rights at the end of the twentieth century. The third one analyzes internal elements that influenced. The last part analyzes some specific cases on the link between foreign policy and human rights in the Mexican case.

KEY WORDS: Human rights, foreign policy, Mexico, international system, democracy.

\footnotetext{
* Artículo recibido el 10 de enero de 2017 y aceptado el 24 de marzo de 2017.

** Facultad de Economia y Relaciones Internacionales, Universidad Autónoma de Baja California. (rafael.velazquez@ uabc.edu.mx)
} 


\section{SUM ARIO}

1. Introducción

2. Política exterior y derechos humanos: el caso mexicano

3. Cambios en el sistema internacional: del fin de la Guerra Fría a la globalización

4. Cambios internos en México: la apertura democrática y el nuevo modelo de desarroIlo económico

5. La política exterior mexicana en materia de derechos humanos a partir de la alternancia del poder

6. Conclusiones

\section{Introducción}

Por mucho tiempo, los derechos humanos no fueron un asunto prioritario en la agenda de política exterior de México. La ausencia se debía a que los gobiernos priistas buscaban alejar la crítica externa a los excesos antidemocráticos y a la alta represión social. El PRI evitaba promocionar los derechos humanos en el ámbito global para impedir que otros actores externos intervinieran en los asuntos del país. Con el argumento del principio de política exterior de no intervención, México imposibilitó la presencia de organismos promotores de derechos humanos en el territorio nacional.

A finales de la década de los noventa e inicio del siglo xxI, el tema experimentó un significativo giro. A partir de entonces, México incorporó la promoción de la democracia y los derechos humanos como un tema importante en sus vínculos con el exterior. Esa decisión causó sorpresa debido a la negativa del gobierno a someterse al escrutinio internacional en la materia. En este sentido, la pregunta relevante es: ¿qué factores influyeron para que México incluyera en su agenda de política exterior los derechos humanos y la promoción de la democracia?

El objetivo primario de este artículo es responder a ese cuestionamiento. Es necesario conocer las causas que llevaron al país a impulsar un tema 138 que hoy es fundamental en la política internacional. El argumento principal es que dicho cambio se debió a un conjunto de factores tanto externos como internos. Por un lado, el gobierno mexicano estuvo expuesto a presiones internacionales para modificar su política en materia de derechos humanos. Por el otro, cambios políticos y económicos internos incentivaron a México a incluir dicho tema en su política exterior. Este texto plantea que los factores internos tuvieron mucho mayor peso en la adopción de una política activa en derechos humanos. 
El artículo está dividido en cuatro partes. La primera plantea la vinculación entre política exterior y derechos humanos desde una perspectiva conceptual. Asimismo, esta sección describe de manera breve algunos antecedentes del caso mexicano. La segunda parte explica los factores externos que motivaron a México para proyectar una política exterior distinta en materia de derechos individuales. La tercera analiza los elementos internos que incidieron en el cambio de la política mexicana hacia esos derechos. La última parte revisa algunos casos específicos a partir de la alternancia del poder en México.

\section{Política exterior y derechos humanos: el caso mexicano}

La vinculación entre política exterior y derechos humanos ha sido ampliamente estudiada en el mundo y en México. Por ejemplo, en Estados Unidos y en Europa, el tema ha sido de gran atención no sólo para los académicos, sino también para los gobiernos, los organismos internacionales y la sociedad civil. El principal interés ha radicado en explicar el surgimiento de normas e instituciones encargadas de la defensa de los derechos humanos y del impacto que éstas tienen en los diferentes países.

En el caso mexicano, Alejandro Anaya, Ana Covarrubias y Natalia Saltalamacchia han contribuido significativamente en el tema. Sus aportaciones han construido una literatura muy vasta sobre los derechos humanos desde una perspectiva de las relaciones internacionales. Por ejemplo, Anaya ha desarrollado un extenso trabajo en torno a la conducta de México en los diferentes foros internacionales y al establecimiento de una política sobre la materia. ${ }^{1}$ Asimismo, ha realizado evaluaciones sobre la política exterior de México sobre la materia en periodos específicos. Por su parte, Covarrubias y Saltalamacchia han buscado explicar el reciente interés del gobierno mexicano en incluir el tema en su política exterior; han hecho estudios sobre la política del país, especialmente sobre las reformas al marco legal. ${ }^{2}$

Parte de la literatura existente se centra en la diferenciación entre política interna y externa y la relación que ambas tienen respecto a los derechos humanos. ${ }^{3}$ La primera se refiere a las medidas que toma un gobierno para garantizar

\footnotetext{
${ }^{1}$ Anaya, Alejandro, "Actors and Processes in the Generation of Change in the Human Rights Policy of Mexico", en Mónica Serrano, Vesselin Popovski y Nicholas Turner (eds.), Human Rights Regimes in the Americas, Tokyo, United Nations University Press, 2010. AnaYa, Alejandro, El país bajo presión: debatiendo el papel del escrutinio internacional de derechos humanos sobre México, México, CIDE, 2014.

${ }^{2}$ Covarrubias, Ana y Natalia Saltalamacchia, Los derechos humanos y la política exterior de los países de América Latina, México, ITAM - Porrúa, 2011.

${ }^{3}$ Negrin, Alejandro, "Una política exterior de derechos humanos para la democracia", El Cotidiano, núm. 150, 2008.
} 
el respeto a los derechos del hombre en el ámbito interno, así como las normas y las instituciones creadas para atender el asunto. La segunda alude a la posición que adopta un país en el ámbito global en materia de derechos humanos, así como las medidas internas que toma en función de las normas externas y la presión que ejercen los organismos internacionales y otros actores. ${ }^{4}$

La política exterior de derechos humanos se define como el conjunto de decisiones, acciones, objetivos, estrategias, medidas y posiciones que adopta un Estado más allá de sus fronteras sobre esta temática en particular. Ambas políticas, la interna y la externa, están íntimamente entrelazadas porque hoy en día no es posible aislarse de un marco jurídico internacional que vela por el respeto a los derechos del hombre en todos los países. La reforma a la Constitución de 2011 en la materia implicó la apertura del Estado mexicano al derecho internacional. A partir de esa fecha, México incorporó los tratados en esa materia al derecho interno con rango constitucional. Por lo tanto, el Estado está obligado a respetar y hacer cumplir las normas internacionales de derechos humanos en el contexto interno.

A partir del fin de la Segunda Guerra Mundial, los derechos del hombre atrajeron mucha atención, especialmente después de las atrocidades en contra de las poblaciones civiles, como el genocidio de los judíos. Uno de los documentos de mayor relevancia en la materia fue la Declaración Universal de los Derechos Humanos de 1948, en el marco de la Organización de las Naciones Unidas. A partir de ahí, organizaciones internacionales, gobiernos, académicos y sociedad civil se han ocupado de este fenómeno y de promover su respeto.

En el marco de la Guerra Fría, el tema cobró importancia especialmente en las guerras internacionales y los conflictos internos de algunos países. Los genocidios en diferentes naciones, la existencia del apartheid y la segregación racial en otras, los golpes de Estado y los movimientos sociales por mejores condiciones, hicieron que el tema de los derechos humanos adquiriera primacía. Por ello, los Estados y las organizaciones internacionales, de la mano de académicos y sociedad civil, promovieron la construcción de instituciones y

140 normas internacionales que velaran por los derechos de las personas, imponiendo castigos a quienes no cumplieran. Así, surgieron instrumentos tales como la Convención para la Prevención y la Sanción del Delito de Genocidio (1948), la Convención Europea de Derechos Humanos (1950), la Convención sobre el Estatuto de los Refugiados (1951), la Convención Internacional sobre

\footnotetext{
${ }^{4}$ CASLA, Koldo, "El papel de los derechos humanos en la política exterior de y en la Unión Europea: un modelo de análisis", Relaciones Internacionales, núm. 17, 2011.
} 
la Eliminación de todas las Formas de Discriminación Racial (1966), la Convención sobre la eliminación de todas las formas de discriminación contra la mujer (1979), la Convención contra la Tortura y Otros Tratos o Penas Crueles, Inhumanos o Degradantes (1984), la Convención sobre los Derechos del Niño (1989), la Convención Internacional sobre la protección de los derechos de todos los trabajadores migratorios y de sus familiares (1990), la Declaración sobre los defensores de derechos humanos (1999), entre otras muchas. Por otro lado, aparecieron organizaciones promotoras de los derechos humanos tales como la Oficina del Alto Comisionado para los Derechos Humanos de la onU, el Alto Comisionado de Naciones Unidas para los Refugiados (ACNUR), el Consejo de Derechos Humanos de la onu, entre muchos otros. Asimismo, varias organizaciones han creado cortes para la defensa de los derechos humanos, como es el caso de la Corte Interamericana de Derechos Humanos de la OEA. De igual manera, han surgido diversas organizaciones no gubernamentales dedicadas al asunto, como Amnistía Internacional y Human Rights Watch. El objetivo era garantizar que todos los países asuman un compromiso formal por el respeto a la dignidad de las personas y su mejoramiento social.

En el caso mexicano, el tema fue totalmente secundario después de la Segunda Guerra Mundial. Los gobiernos priistas no se preocuparon del asunto porque se enfocaban a los asuntos internos con mayor atención. Como el sistema político era altamente centralizado y represivo frente a los diferentes movimientos sociales, el gobierno buscaba ocultar esa realidad ante los ojos externos. El jefe del Ejecutivo principalmente anteponía el principio de la no intervención para evitar que organizaciones internacionales $\mathrm{u}$ otros gobiernos se entrometieran en los asuntos internos del país. Incluso, México exigía que otros Estados no intervinieran en otros países. Un caso particular fue Cuba. Luego de la revolución castrista a finales de la década de los cincuenta, México fue un fiel defensor de la no intervención en la isla. En la práctica, parecía que México defendía a Cuba para evitar que otros gobiernos u organismos internacionales pudieran interferir en sus asuntos internos. El objetivo era ocultar la violación a los derechos humanos que el gobierno priista ejercía en su represión a los diferentes sectores sociales.

Un evento que resultó un hito fue la masacre a estudiantes mexicanos en octubre de 1968. El ejército asesinó a cientos de jóvenes que protestaban por falta de democracia y por la represión del gobierno frente a los opositores. El presidente Gustavo Díaz Ordaz decidió aplastar el movimiento porque en pocos días iniciaban en la Ciudad de México los juegos olímpicos. El PRI no quería mostrar al mundo la falta de cohesión social que existía en el país. Debido 
al alto control del gobierno sobre los medios de comunicación, el evento fue prácticamente desconocido en México y el exterior. Sin embargo, a partir de ese momento la sociedad mexicana empezó a demandar mayores libertades y mejores condiciones de vida.

En los años setenta, aparecieron en el país movimientos guerrilleros que luchaban por mejores condiciones de vida para la población más vulnerable. En lugar de abrir el sistema, el gobierno priista optó por lanzar una guerra sucia en contra de los alzados. La represión en los años setenta fue implacable, pero al exterior poco se sabía por el control de los medios de comunicación y por la cerrazón del gobierno al escrutinio internacional. A finales de los años setenta y principios de los ochenta, el país experimentó profundas crisis económicas que impactaron negativamente en la calidad de vida de los mexicanos. De la misma manera, en diferentes partes del país se presentaron fraudes electorales para mantener al PRI en el poder. A pesar de todo ello, el gobierno mantuvo una política cerrada frente al escrutinio internacional en materia de derechos humanos. Fue hasta finales de la década de los años ochenta e inicios de los noventa cuando el gobierno mexicano empezó a mostrar una apertura débil al tema de los derechos humanos.

\section{Cambios en el sistema internacional: del fin de la Guerra Fría a la globalización}

Los cambios externos e internos de finales del siglo pasado y principios del presente impactaron de manera positiva para incluir el tema de los derechos humanos en la agenda de la política exterior de México. Por ello, es indispensable describir de manera general aquellos elementos de orden local y global que posibilitaron el fenómeno descrito en este trabajo. Por ello, este apartado reseña los factores externos de mayor impacto que tuvieron influencia en la adopción de una política internacional más favorable a los derechos humanos en México.

En parte, los cambios recientes en el sistema internacional explican el interés de México por promocionar los derechos humanos en el plano global. Por un lado, el fin de la Guerra Fría representó un importante cambio en el equilibrio del poder del sistema internacional. En efecto, la desaparición de la Unión Soviética y del bloque socialista modificó la correlación de fuerzas en el contexto internacional. Estados Unidos quedó como la principal superpotencia económica-militar. Pero otros actores empezaron a competir fuertemente con Estados Unidos en el ámbito económico, como la Unión Europea, Japón y China. En consecuencia, el sistema internacional contemporáneo está caracteriza- 
do por una unipolaridad desde el punto de vista militar y una multipolaridad en la esfera económica.

Con el fin de la Guerra Fría, los objetivos de política exterior de los principales actores cambiaron significativamente. Entre 1945 y 1989, el énfasis estuvo en evitar la expansión comunista, promover el desarme nuclear y mantener las esferas de influencia por parte de cada potencia. A partir de 1989, los principales objetivos se han orientado a una mayor integración económica, una lucha contra las principales amenazas (terrorismo y narcotráfico), la búsqueda de un equilibrio financiero, el enfrentamiento al cambio climático, reducción de la pobreza en el mundo, defensa de los derechos humanos, entre otros.

El cambio de equilibrio del poder en el sistema internacional y los nuevos objetivos de política exterior de los principales actores fueron favorables para que otros temas aumentaran su presencia en la agenda de la política mundial. Los objetivos de política exterior más importantes estaban enfocados en cuestiones de seguridad y de mantenimiento de sus zonas de influencia. Por lo tanto, la política internacional se concentraba principalmente entre las superpotencias y sus respectivos aliados. Con esta lógica fuera del escenario y con la ayuda del proceso de globalización, otros temas empezaron a tener una mayor visibilidad en el contexto internacional. Desde esta perspectiva, los derechos humanos empezaron a tener una mayor relevancia en la nueva dinámica internacional. La sociedad internacional se preocupaba principalmente por los derechos de los más vulnerables, como niños, ancianos, mujeres, indígenas; pero también había interés por proteger a los migrantes, refugiados, las minorías raciales, las personas con preferencias sexuales diferentes, entre otros.

Por otro lado, la globalización económica y una mayor interdependencia favorecieron la presencia de los derechos humanos en la agenda de la política internacional. Hoy en día es prácticamente imposible que un país se aísle del contexto externo. La globalización ha ayudado a internacionalizar la política de protección a los derechos del hombre. Asimismo, los procesos de integración económica y liberalización comercial que se han puesto de moda en los últimos años han presionado para que exista un mayor respeto a los derechos humanos y una garantía de que la democracia debe prevalecer. En la negociación de algunos acuerdos de libre comercio, muchos actores condicionan a los países a que garanticen la defensa de esos valores como condición sine qua non para alcanzar un mayor nivel de integración. En el caso de México, la firma del Tratado de Libre Comercio con Estados Unidos y Canadá que entró en vigor en 1994 facilitó una mayor presencia de los derechos individuales 
en la agenda internacional ${ }^{5}$ puesto que la sociedad estadounidense criticaba a México porque su gobierno no respetaba los derechos humanos. Por ello, algunos legisladores estadounidenses condicionaban un compromiso más fuerte por parte de México para aprobar dicho acuerdo.

Por mucho tiempo, México se mantuvo cerrado al comercio exterior al adoptar un modelo sustitutivo de importaciones. Una consecuencia de esa estructura era, también, un aislacionismo en materia de política exterior. Por lo tanto, el gobierno mexicano se mantenía cerrado de igual manera al escrutinio externo en materia de derechos civiles. Con la liberalización económica, el país optaba por un capitalismo más agresivo. Era claro que, para este modo de producción, la democracia y el respeto a las libertades eran fundamentales para su mantenimiento. Por ello, la promoción de los derechos individuales resultaba vital para entrar en la nueva dinámica internacional basada en el modelo neoliberal.

Como se puede observar, las transformaciones recientes en el sistema internacional y el cambio en los objetivos centrales de los actores han facilitado e incentivado una mayor presencia de los derechos del hombre en la agenda de la política internacional. La globalización impide el aislacionismo y, por lo tanto, es difícil que cualquier Estado oculte las violaciones a esos derechos. Finalmente, los procesos de integración ayudan a garantizar la democracia y la protección a los derechos de los individuos porque una condición para firmar acuerdos de libre comercio, por ejemplo, es la garantía del respeto a las prerrogativas mínimas del hombre. Además, en algunos casos, los procesos de integración consolidados, como por ejemplo la Unión Europea, abren la posibilidad de una mayor supervisión en la materia a la hora de firmar acuerdos con otros países.

\section{Cambios internos en México: la apertura democrática y el nuevo modelo de desarrollo económico}

México experimentó profundos cambios políticos y económicos a partir de finales del siglo xx y principios del xxi. Obviamente, esa nueva realidad tendría un importante impacto para que el gobierno mexicano tuviera interés de proyectar una política exterior más favorable a los derechos del hombre. Por ello, este apartado busca explicar dichas transformaciones. La primera parte analiza la apertura democrática que el país experimentó en ese periodo y la segunda

\footnotetext{
${ }^{5}$ Anaya, Alejandro, "Libre comercio y política exterior en materia de derechos humanos en América del Norte. Una evaluación de la tesis del derrame (spillover)", Espiral, Estudios sobre Estado y Sociedad, vol. 16, núm. 46, 2009.
} 
explica cómo el cambio en el modelo de desarrollo económico impactó sobre la materia de estudio de este ensayo.

En los últimos años del siglo xx, México experimentó profundas transformaciones en su sistema político. Anteriormente el régimen presidencialista fuerte no daba mucho margen de acción para la promoción de los derechos humanos en el contexto externo. Como el jefe del Ejecutivo ejercía el poder prácticamente de manera absoluta, no había mucho interés de abrir el país a la democracia y el tema de los derechos humanos era completamente secundario. Los excesos antidemocráticos al interior hacían que las administraciones priistas mostraran cerrazón ante el escrutinio internacional. Además, el Ejecutivo prácticamente mantenía un monopolio de la política exterior y no tenía interés en promocionar los derechos humanos. Como el sistema político era autoritario y represivo, el PRI quería ocultar esa realidad ante los ojos externos.

Muchos presidentes priistas habían llegado al poder a través de procesos poco democráticos. Por ello, utilizaban a la política exterior como un mecanismo para ganar legitimidad democrática. La estrategia era ser crítico frente a Estados Unidos y buscar un acercamiento estrecho con América Latina para construir consensos internos. México mantuvo una relación amistosa incluso con gobiernos de izquierda, como Cuba y Nicaragua, con el propósito de satisfacer los intereses de los grupos nacionalistas. Sin embargo, la promoción de los derechos humanos no estaba en la lista de esas estrategias. Abrirse al escrutinio externo resultaba contradictorio puesto que al interior existía falta de democracia y un compromiso débil respecto a los derechos humanos.

Un acontecimiento que marcó un antes y un después fue la elección presidencial de 1988. En esa ocasión, el PRI volvió a ganar la elección pero prácticamente tuvo que recurrir a un fraude para logarlo. En consecuencia, el presidente Carlos Salinas de Gortari tuvo que promover cambios en la esfera democrática para legitimar su administración y satisfacer a la creciente oposición. El sistema electoral mexicano experimentó importantes reformas políticas para abrir el sistema a una sociedad sedienta de democracia.

El gobierno creó importantes instituciones y modificó las leyes para una mayor apertura democrática, como fue el caso del Ombudsman, dependiente de la Secretaría de Gobernación y la reforma de las leyes electorales. Sin embargo, el PRI mantuvo la hegemonía política y el dominio de la agenda nacional. El presidente Salinas solamente aceptó que la oposición ganara algunos estados de la república, pero solamente reconoció el triunfo del partido conservador Acción Nacional (PAN). La izquierda prácticamente estuvo vetada del poder. 
Los cambios más importantes en materia de derechos humanos vinieron a mediados y finales de la década de los noventa. El siguiente presidente, Ernesto Zedillo Ponce de León, impulsó una mayor apertura democrática y abrió a México, por primera vez, al escrutinio internacional. Diversas reformas electorales aprobadas en su administración crearon un ambiente más propicio para la defensa de las libertades en México. Por ejemplo, Zedillo ciudadanizó el IFE e impuso medidas más estrictas para evitar los fraudes electorales. Como una novedad en el sistema político, México aceptó la presencia de observadores externos para los procesos políticos (por ejemplo, las elecciones) y permitió la entrada al país de relatores de organizaciones internacionales en materia de derechos del hombre.

El gobierno de Zedillo fue el que más apertura mostró en el ámbito político. Por ejemplo, en 1997 aceptó y reconoció el triunfo de la oposición en las elecciones para diputados federales. Por primera vez en la historia, el PRI no tenía mayoría absoluta en una de las cámaras legislativas. La apertura democrática de Zedillo se confirmó tres años después cuando reconoció el triunfo del PAN en las elecciones presidenciales. La llegada de Vicente Fox, el primer presidente no priista, fue un acontecimiento de suma importancia para el sistema político y representó una de las mayores transformaciones políticas del país. A partir de ese triunfo, el tema de las libertades individuales empezó a tener una gran importancia en la agenda de la política exterior de México.

Con la desaparición del régimen autoritario y centralista priista a finales de los años noventa, los actores no gubernamentales empezaron a presionar para una mayor apertura en el tema de los derechos humanos y el gobierno empezó a mostrar un incipiente interés en el asunto. Obviamente, en el sexenio de Zedillo existieron destacadas organizaciones civiles promotoras de las libertades de los individuos, pero la alternancia política permitió una mayor apertura del gobierno hacia esos actores.

Un argumento de este ensayo es que el cambio en el modelo de desarrollo económico también fue un factor determinante para que la política exterior de

146 México incluyera en su agenda a los derechos humanos como un tema prioritario. Anteriormente, el modelo de desarrollo económico de México estaba basado en el esquema sustitutivo de importaciones. Una economía cerrada daba pocas oportunidades para que el tema fuera importante. Sin embargo, a partir de mediados de los años ochenta, México inició un proceso de apertura económica caracterizado por la liberalización del comercio exterior y la atracción de inversiones externas. Las causas inmediatas de este giro se encuentran en la crisis financiera de principios de los años ochenta, causada por la baja del 
precio internacional del petróleo y del aumento de las tasas de interés respecto a la deuda contratada por los países en vías de desarrollo.

Con la nueva política económica, los derechos humanos tuvieron una mayor oportunidad para incrementar su presencia y visibilidad en los asuntos exteriores de México. Los ejemplos más representativos tuvieron lugar en el sexenio de Salinas de Gortari y fueron la negociación del Tratado de Libre Comercio con América del Norte y el ingreso de México a la Organización para la Cooperación y el Desarrollo Económico (ocDE). En el primer caso, varios sectores sociales de Estados Unidos criticaban la falta de respeto a los derechos humanos en México. Para no arriesgar un posible rechazo del congreso de Estados Unidos, el gobierno de Salinas de Gortari creó la Comisión Nacional de Derechos Humanos (CNDH) en 1990.

Este organismo está a cargo de velar por los derechos humanos en México. Sin embargo, la génesis de esta institución está ligada al cambio en el modelo de desarrollo económico. La administración salinista quiso mostrar una cara de preocupación por el tema para garantizar la aprobación del TLCAN, una de las principales iniciativas de su sexenio. La creación de la CNDH fue un hito en la historia de los derechos humanos en México. Sin embargo, su origen fue producto de dos factores: en primer lugar, la cNDH fue una concesión del gobierno de Salinas luego del fraude electoral de la elección de 1988. Su objetivo real era ganar legitimidad democrática. En segundo lugar, la CNDH se creó para evitar la crítica externa y garantizar la aprobación del TLCAN. En ambos casos, no había un legítimo interés por la defensa de los derechos humanos. Además, la comisión dependía de la Secretaría de Gobernación, lo que minaba su autonomía. La primera gran contradicción del salinismo fue la represión a la insurgencia zapatista en 1994. Por un lado, su administración se comprometía a favor de las libertades de los individuos y, por el otro, aplastaba los movimientos sociales que buscaban mejorar las condiciones de las clases más necesitadas.

En el segundo caso, la administración salinista solicitó ingresar a la OCDE a mediados de los años noventa. Para ello, era fundamental que México mostrara avances importantes en materia de respeto a los derechos humanos, una de las principales exigencias de ese organismo para ganar su membrecía. Por ello, la administración de Salinas buscó proyectar una imagen en donde la dignidad de las personas fuera una prioridad para México. En síntesis, la firma del TLCAN y uno de los acuerdos paralelos, como el acuerdo laboral y el ingreso a la oCDE, representaban dos elementos que pondrían a México en la senda de la modernización, una prioridad incuestionable para Salinas de Gortari. Para el logro de estos objetivos, un compromiso más fuerte con los derechos humanos era esencial. 
Otro ejemplo que demuestra el argumento es la negociación del Tratado de Libre Comercio con la Unión Europea (TLCUEM) que se presentó a mediados y finales de la década de los noventa. En este caso, Bruselas exigió a México la inclusión de la cláusula democrática para garantizar el compromiso de procesos políticos abiertos y el respeto de los derechos humanos. Al principio, el gobierno mexicano se mostró renuente al respecto. Aceptó incluir la cláusula pero con una reserva que garantizara la no intervención en los asuntos internos de México. Sin embargo, la Unión Europea no aceptó esa posición y prácticamente le exigió al país la firma de tal instrumento sin ninguna reserva.

A regañadientes, el gobierno mexicano tuvo que aceptar la exigencia europea. Lo anterior muestra que los procesos de integración económica están ligados a la democracia y al respeto de la dignidad de las personas. A final de cuentas, la administración zedillista tuvo que aceptar las condiciones de la Unión Europea. Sin embargo, durante su sexenio hubo también dos contradicciones: el no reconocimiento de la autodeterminación de los pueblos originarios indígenas y la no puesta en vigor del Convenio 169 de la orт que se refiere a los pueblos indígenas y tribales en países independientes.

\section{La política exterior mexicana en materia de derechos humanos a partir de la alternancia del poder}

En el año 2000, el PRI perdió la elección presidencial después de más de setenta años de predominio. La llegada de un partido distinto significó un cambio profundo en el sistema político mexicano. Ello implicó también un giro en el tema de los derechos humanos. Como el nuevo presidente Vicente Fox había llegado a través de un proceso transparente y democrático, su gobierno podía abiertamente modificar la tendencia priista e iniciar una verdadera promoción de la democracia y el respeto a los derechos humanos. Efectivamente así se hizo. Desde un principio, el presidente Vicente Fox planteó en su proyecto de política exterior la inclusión de los derechos humanos y la democracia como

148 un tema prioritario en la agenda. El objetivo era anclar esos temas en el sistema político para las generaciones futuras. Es decir, los cambios en esa materia adoptados por la nueva administración crearían compromisos ineludibles para México en el futuro. Por lo tanto, no importaría qué partido gobernara el país. Ningún gobierno podría dar marcha atrás a los cambios y avances en materia democrática y de derechos humanos.

Tanto en el sexenio de Fox y Calderón, hubo casos significativos relacionados con la materia de estudio de este escrito. Algunos le dieron a México prestigio, pero otros representaron graves problemas para la política exterior 
mexicana. A continuación se abordan los casos más representativos en ambas administraciones.

Desde el principio de su administración, Vicente Fox quería proyectar una política exterior que reflejara la promoción de la democracia y los derechos humanos. El "bono democrático" le daba la autoridad necesaria y el margen de acción para ello. Desde su campaña, y en los primeros meses de su gobierno, Fox planteó como un objetivo prioritario para la política exterior la defensa de ambos temas. En el Plan Nacional de Desarrollo 2001-2006, el tema fue incluido por primera vez. Parecía que el nuevo presidente quería cambiar la política tradicional priista en la materia y presentar una propia. ${ }^{6}$ Cuba se convirtió rápidamente en el objetivo inmediato. Por mucho tiempo, el gobierno priista había adoptado una posición amistosa hacia Fidel Castro. Fox quería cambiar esa política priista porque consideraba que en la isla no había procesos democráticos y no se respetaban los derechos humanos de los disidentes políticos.

En 2002, Vicente Fox hizo una visita oficial a Cuba. En esa ocasión, el presidente mexicano se reunió con la oposición castrista. ${ }^{7}$ Rápidamente, el gobierno de la isla consideró el evento como un acto no amistoso y una intervención en los asuntos de Cuba. A partir de ahí, la relación empezó a tomar tintes de tensión. La administración de Fox consideraba que estaba haciendo lo correcto porque su objetivo era simplemente promover la democracia y los derechos humanos en la isla. Sin embargo, el asunto que marcó un antes y después en la relación bilateral fue la reunión en Ginebra de la Comisión de Derechos Humanos de la onu en el 2002.

En esa ocasión, Estados Unidos promovió una condena al régimen de Castro por no respetar los derechos humanos y proponía la visita de un relator a la isla. ${ }^{8}$ Obviamente, Cuba no estaba a favor de esa resolución y buscó apoyo con varios países para que no fuera aprobada. Al gobierno de Castro le interesaba tener el apoyo de México porque en el pasado había sido su protector y, además, buscaba no estar aislado. Sin embargo, México votó a favor de la propuesta con base en su nueva política internacional de promoción de los derechos humanos. El hecho vino a complicar la relación entre México y Cuba.

Pero el acontecimiento que puso a la relación al borde del rompimiento fue la asistencia de Fidel Castro a una reunión de la onu en Monterrey, México. Antes

\footnotetext{
${ }^{6}$ Velázouez, Rafael, "El proyecto de política exterior de Vicente Fox: ¿Continuidad o cambio?", en Rafael Velázquez (coord.), La política exterior de México bajo un régimen democrático ¿Cambio o continuidad?, México, Plaza y Valdés, 2002.

7 OJeDA, Mario, "Vicente Fox: El rompimiento de facto con Cuba", Foro Internacional, vol. 47, núm. 4, 2007.

${ }^{8}$ Pascoe, Ricardo, En el filo. Historia de una crisis diplomática. Cuba 2001-2002, México, Ediciones Sin Nombre, 2004.
} 
de llegar, Vicente Fox le pidió a Castro en una llamada telefónica asistir a la reunión e irse inmediatamente después de terminar su discurso para no complicar la asistencia del presidente de Estados Unidos, George Bush. De manera disciplinada, Castro atendió la sugerencia pero al salir de México informó a la prensa que se tenía que ir de inmediato porque así se lo habían solicitado. De inmediato, los reporteros le preguntaron al general quién le había pedido irse con anticipación. Castro eludió la respuesta y el asunto quedó ahí. ${ }^{9}$ Sin embargo, más tarde y después del voto de México en Ginebra, Castro reveló, en represalia, la conversación telefónica privada que había tenido con Vicente Fox en donde le pedía llegar a la reunión e irse de inmediato. El incidente fue conocido como el "comes y te vas", y causó una grave crisis diplomática entre ambos países.

El asunto no quedó ahí. Dos años más tarde, México volvió a votar en contra de Cuba en la Comisión de Derechos Humanos de la onu. Luego de un discurso de Castro en donde criticaba la política internacional de México, el presidente Fox decidió expulsar al embajador cubano y reducir el nivel de la relación a "encargados de negocios". Prácticamente fue una ruptura de facto. ${ }^{10}$ De inmediato, la opinión pública mexicana criticó fuertemente la medida $\mathrm{y}$ Cuba buscó desprestigiar a México. Por primera vez, la relación con la isla estaba al borde del rompimiento y Fox se encontraba frente a un problema interno por las críticas que recibió por tomar esa medida. Más adelante, el asunto se resolvió cuando ambos gobiernos volvieron a instalar a sus embajadores.

Era claro que el origen del conflicto con Cuba estaba en la nueva política de promoción de la democracia y los derechos humanos del nuevo presidente; pero también es necesario reconocer otros dos factores externos. El primero es Estados Unidos. Desde la llegada de Fox a la presidencia, Washington había presionado a México para modificar su política hacia Cuba. Estados Unidos no estaba totalmente de acuerdo con el apoyo priista hacia Castro y buscaba que México lo modificara. ${ }^{11} \mathrm{Al}$ llegar Bush a la presidencia en el 2001, la relación entre ambos presidentes fue cordial y el texano presionaba a Fox para reformar la política mexicana hacia Cuba.

150 El segundo factor fue Jorge Castañeda, el primer secretario de Relaciones Exteriores del gabinete de Vicente Fox. Es posible que el canciller mexicano tuviera una aversión personal en contra del régimen comunista de Castro. No hay duda que Castañeda fue uno de los principales involucrados en las decisiones frente a Cuba puesto que ejercía mucha influencia en el presidente Fox.

\footnotetext{
${ }^{9}$ Petrich, Blanche, "Orilló Fox a Castro a dejar la cumbre; Bush llegó más tarde", La Jornada, 22 de marzo, 2002.

${ }^{10}$ OJeDA, Mario, "Vicente Fox: El rompimiento de facto con Cuba", Foro Internacional, vol. 47, núm. 4, 2007.

"Pascoe, Ricardo, En el filo. Historia de una crisis diplomática. Cuba 2001-2002, México, Ediciones Sin Nombre, 2004.
} 
De hecho, el entonces secretario de Relaciones Exteriores había sido uno de los principales asesores de Fox durante la campaña y uno de los funcionarios más influyentes al inicio de la administración panista.

En resumen, Fox buscó con Cuba hacer realidad su objetivo de promover la democracia y los derechos humanos. Sin embargo, se enfrentó a una crisis diplomática que desprestigió a México en la región latinoamericana debido a los episodios del "comes y te vas" y a la expulsión del embajador cubano. Parecía que la intención era la correcta, pero falló la operación diplomática.

Con la llegada de Felipe Calderón a la presidencia en 2006, el tema se mantuvo como una prioridad para la agenda de política exterior, pero con un matiz distinto. La administración calderonista buscó mantener la misma tendencia respecto al asunto, pero buscando evitar involucrarse en crisis, como lo hizo Vicente Fox. Pero Calderón tenía un contexto muy diferente al de su antecesor. En primer lugar, el nuevo presidente había ganado la elección con un margen muy estrecho de votos y la oposición de izquierda no lo reconoció como presidente legítimo.

En otras palabras, Calderón llegó al poder con una legitimidad altamente cuestionada. En segundo lugar, el país estaba inmerso en una violencia generalizada debido a la lucha en contra del narcotráfico. Miles de personas eran asesinadas cada día y el ambiente de inseguridad prevalecía en todo el país. Por estas razones, Calderón tuvo que mantener el tema de los derechos humanos desde una perspectiva más discreta.

Para tener ventajas frente a los carteles del narcotráfico y tratar de reducir la violencia, el gobierno calderonista buscó apoyo en Estados Unidos. Después de una reunión con el presidente Bush en la ciudad de Mérida, surgió una iniciativa para que Washington pudiera otorgar a México un financiamiento para fortalecer sus capacidades en la lucha contra el tráfico ilegal de drogas. La idea fue conocida más tarde como la Iniciativa Mérida. Sin embargo, los legisladores estadounidenses no estaban totalmente de acuerdo en ofrecer esa ayuda porque consideraban que el Ejército mexicano y las policías violaban los derechos humanos de manera sistemática. Entonces, el congreso estadounidense buscó imponer condiciones a México para poder otorgar el financiamiento comprometido en la Iniciativa Mérida. ${ }^{12}$ Dos requisitos eran un mayor compromiso en materia de derechos humanos y la creación de tribunales civiles para enjuiciar a militares que hayan cometido actos en contra de la dignidad de las personas.

\footnotetext{
12 "Condiciona EU fondos para Iniciativa Mérida", El Universal, 18 de mayo, 2008.
} 
En un principio, la opinión pública mexicana estaba en contra de la Iniciativa Mérida porque existía la percepción de que era una forma de intervenir por parte de Estados Unidos. ${ }^{13}$ El ejército mexicano no estaba de acuerdo en la existencia de los tribunales civiles y los legisladores mexicanos no aceptaban la imposición de condiciones en materia de derechos humanos porque lo consideraban una intervención a los asuntos internos de México. A final de cuentas, la iniciativa fue aprobada con algunos matices para que los actores (tanto gubernamentales como no gubernamentales) de ambos lados de la frontera estuvieran de acuerdo.

La Iniciativa Mérida fue un logro diplomático de la política exterior de México porque hizo que Estados Unidos reconociera abiertamente su corresponsabilidad en el asunto y ofreciera apoyo económico. Sin embargo, el mecanismo no fue suficiente para reducir la violencia y hacer una defensa más efectiva de los derechos humanos en México. Además, la Iniciativa Mérida, en su parte de evaluaciones, influyó para que el sistema jurídico penal mexicano cambiara. Tal fue el caso del Código Penal.

Como Calderón mantuvo la tendencia de promover el tema, ahora la estrategia se centró hacia dentro. En este sentido, Calderón presentó una iniciativa para una reforma constitucional muy amplia en materia de derechos humanos. ${ }^{14}$ La propuesta fue aprobada por los legisladores y ahora la Carta Magna busca garantizar una cultura de respeto a los derechos humanos entre la sociedad y el gobierno. La reforma promueve un amplio espectro de libertades sociales, económicas y políticas para los mexicanos. El objetivo es cambiar la tendencia prevaleciente para fomentar una práctica más estricta en la materia.

Con esta reforma constitucional, con amplio marco jurídico que establece normas específicas y con un número amplio de instituciones encargadas del tema, ya existen suficientes incentivos para moldear el comportamiento de las autoridades y de la sociedad hacia un mayor respeto hacia las personas.

Uno de los puntos más destacados de la reforma constitucional en materia de política exterior fue la inclusión de un nuevo principio en el artículo 89, fracción x. Desde finales de los años ochenta, el congreso mexicano aprobó una modificación a ese artículo para introducir siete principios tradicionales de

\footnotetext{
${ }^{13}$ Chabat, JoRge, "La Iniciativa Mérida y la relación México-Estados. Unidos: en busca de la confianza perdida", en Rafael Velázquez y Juan Pablo Prado (coords.), La Iniciativa Mérida: ¿Nuevo paradigma de cooperación entre México y Estados Unidos en seguridad?, México, UNAM - BUAP, 2009.

${ }^{14}$ Saltalamacchia, Natalia y Covarrubias, Ana (2011), "La dimensión internacional de la reforma de derechos humanos: antecedentes históricos", en Miguel Carbonell y Pedro Salazar (coords.), La reforma constitucional de derechos humanos: un nuevo paradigma, México, unam, 2011.
} 
las relaciones exteriores de México: la no intervención, la autodeterminación de los pueblos, la igualdad jurídica de los Estados, la solución pacífica de las controversias, la cooperación internacional para el desarrollo, la proscripción de la amenaza o el uso de la fuerza en las relaciones internacionales y la lucha por la paz y la seguridad internacionales. Con la reforma de 2011, un octavo principio fue incluido: la protección y promoción de los derechos humanos. Con esta modificación del marco jurídico, el gobierno mexicano está obligado en los hechos a impulsar fuera de México los derechos humanos. Esta medida busca anclar en México una política exterior que favorezca los derechos humanos ante la comunidad internacional, ya sean otros países, organismos internacionales y diferentes foros.

En general, México ha tenido avances significativos en la relación entre política exterior y derechos humanos. En su momento, la Corte Suprema de la Nación igualó los tratados internacionales en materia de derechos humanos a nivel constitucional. Es decir, los diferentes instrumentos internacionales en la materia tienen el mismo valor que la Carta Magna. Además, México se ha comprometido a aceptar las resoluciones de la Corte Interamericana de Derechos Humanos (CIDH) de la OEA, a través del principio de convencionalidad y control de convencionalidad. Con esta medida, los mexicanos que consideren que sus derechos humanos han sido violados y las instancias internas no han resuelto el asunto de manera satisfactoria pueden de manera directa recurrir a la CIDH para encontrar una solución adecuada.

De la misma manera, México ha firmado un número amplio de convenciones sobre la materia y se ha convertido en promotor de muchas de ellas. Entre las más contemporáneas y destacadas están: el Pacto Internacional de Derechos Civiles y Políticos, destinados a abolir la pena de muerte, la Convención contra la Tortura y otros Tratos o Penas Crueles Inhumanos o Degradantes, el Protocolo sobre el Estatuto de los Refugiados, la Convención sobre la Eliminación de todas las formas de Discriminación contra la Mujer, la Convención sobre los Derechos del Niño, el Convenio constitutivo del fondo para el Desarrollo de los Pueblos Indígenas de América Latina y el Caribe, la Convención Internacional sobre la Eliminación de todas las formas de Discriminación Racial, la Convención sobre la Imprescriptibilidad de los Crímenes de Guerra y de los crímenes de Lesa Humanidad, la Convención Internacional sobre la Protección de los Derechos de todos los Trabajadores Migratorios y de sus Familiares, entre muchas otras. Además, México ha tenido una destacada labor y presencia en los diferentes organismos internacionales. Por ejemplo, el país presidió el Consejo de Derechos Humanos de la ONU en su primer año de existencia (2006). 
México ha mantenido una tendencia favorable a los derechos humanos en el plano global y ha puesto el tema como una prioridad en la agenda de política exterior al inicio del siglo xxI. ${ }^{15}$ Esta actitud le ha dado al país un cierto prestigio en el tema. Sin embargo, en los últimos años se han presentado en el contexto interno diferentes violaciones a los derechos humanos que han sido objeto de denuncias en los organismos internacionales, así como las recomendaciones que éstos han emitido en contra de México. Además, las acciones que México ha llevado a cabo en esta materia le han acarreado algunos problemas diplomáticos, como fue el caso concreto de Cuba.

En otras palabras, la inclusión de los derechos humanos en la agenda de la política exterior no garantiza que los gobiernos respeten cabalmente esas prerrogativas. En el caso de México, en los últimos años ha existido una política de promoción amplia sobre el tema en el plano externo. Pero en el contexto interno han existido casos de violación grave a los derechos humanos. Ejemplo de ello han sido las masacres en diversas partes del país (Aguas Blancas en 1995, Acteal en 1997, San Fernando en 2011, Tlatlaya en 2014, Ayotzinapa en el mismo año); los feminicidios en Ciudad Juárez y otros estados de la República; la violación a los derechos humanos por parte del Ejército a la población civil en la lucha contra el narcotráfico; la constante tortura a los detenidos; la extorsión a migrantes centroamericanos por parte de autoridades migratorias, entre muchos otros ejemplos.

\section{Conclusiones}

El presente texto ha mostrado amplia evidencia de que los factores externos (fin de la Guerra Fría y globalización) y los internos (apertura democrática y cambio del modelo de desarrollo económico) son los elementos que explican el interés de México por plantear una política exterior favorable a los derechos humanos. Sin embargo, también es justo reconocer que los presidentes mexicanos (Salinas, Zedillo, Fox y Calderón) desempeñaron un papel destacado en

154 el asunto. En el caso de los dos priistas, su compromiso con los procesos de integración comercial y los necesarios cambios políticos permitió una mayor apertura de México hacia el tema.

\footnotetext{
${ }^{15}$ Negrin, Alejandro, "Una política exterior de derechos humanos para la democracia", El Cotidiano, núm. 150, 2008.
} 
En cambio, para los dos panistas, la ideología y sus preferencias personales a favor de las personas fueron la causa principal por ese interés. No obstante, este texto plantea que los factores internos tuvieron un mayor peso debido a que en el caso mexicano era imposible pensar en cambios importantes en la materia sin reformas políticas significativas y sin un modelo de desarrollo económico que garantizara un mejor nivel de vida para la población y así los derechos económicos y sociales fueran respetados.

La inclusión de los derechos humanos como un tema prioritario en la agenda de política exterior de México no ocurrió a partir de la llegada del PAN al poder en el año 2000. El cambió surgió en los años noventa, como producto del nuevo modelo de desarrollo económico y de la apertura democrática que el país experimentó a finales del siglo xx. Efectivamente, la entrada del partido conservador al poder amplió y fortaleció esa tendencia. Para los políticos conservadores, especialmente Vicente Fox y Felipe Calderón, el tema de los derechos humanos era prioritario por razones ideológicas fundadas en sus preferencias personales y en la plataforma política del PAN. En consecuencia, ambas administraciones panistas buscaron promocionar en el exterior la democracia y el respeto a los derechos humanos. Fox y Calderón querían garantizar que la democracia y los derechos humanos se anclaran en el sistema político mexicano sin importar que otro partido político llegara en el futuro.

El tema de los derechos humanos está íntimamente ligado a los procesos democráticos. A mayor apertura, habría más posibilidades de que se respetaran. La democracia no garantiza una política favorable para los derechos humanos, pero la facilita. De igual manera, este fenómeno está vinculado estrechamente con los procesos de integración económica. Como los países desarrollados exigen el respeto a los derechos humanos como condición para la firma de acuerdos de libre comercio, entonces las naciones menos desarrolladas se ven obligadas a adoptar una política más comprometida con ese asunto. Fue el caso de México particularmente en los casos de la firma de los tratados con América del Norte y con la Unión Europea.

En materia de política exterior, México ha tomado los pasos necesarios para la defensa y promoción de los derechos humanos. Sin embargo, hace falta mucho trabajo para consolidar una política de respeto a la dignidad de las personas en el ámbito interno. En México, existen instituciones encargadas de velar por esos derechos y crear leyes fuertes para castigar sus violaciones. Sin embargo, el problema radica en el personal operativo (policías en los tres niveles de gobierno, soldados, oficiales de migración, jueces). Sin una real transformación institucional y una mayor capacitación de los cuadros 
encargados del asunto, el tema de los derechos humanos seguirá siendo una materia pendiente.

A principios del siglo xxi, los retos de México de cara a los derechos humanos en materia internacional son amplios y variados. En primer lugar, el país debe ser consistente entre su política exterior de promoción de derechos humanos y su actuación en el ámbito interno. Es totalmente contradictorio que en los diferentes foros internacionales el gobierno mexicano sea un actor global responsable y que, al mismo tiempo, existan serias violaciones a la dignidad de los ciudadanos. En segundo lugar, el gobierno mexicano tiene que hacer una reforma profunda en las instituciones encargadas de velar por esos derechos. Es necesaria una mayor capacitación en materia de derechos humanos para policías, soldados, oficiales de migración y todo personal involucrado.

Asimismo, el marco jurídico mexicano se debe adecuar a los nuevos ordenamientos internacionales y proyectar un compromiso más fuerte para cumplir con las resoluciones de los diferentes organismos internacionales. Finalmente, México debe promover la gobernanza en materia de derechos humanos. Es decir, el gobierno debe coordinar la construcción de políticas públicas entre los diferentes actores públicos, sociales y privados para una mayor promoción de los derechos humanos en el ámbito interno y externo. Solamente así, México podrá superar los retos que se presenten a corto y mediano plazo.

\section{Bibliografía}

Anaya, Alejandro, "Libre comercio y política exterior en materia de derechos humanos en América del Norte. Una evaluación de la tesis del derrame (spillover)”, Espiral, Estudios sobre Estado y Sociedad, vol. 16, núm. 46, 2009.

Anaya, Alejandro, "Actors and Processes in the Generation of Change in the Human Rights Policy of Mexico”, en Mónica Serrano, Vesselin Popovski y Nicholas Turner (eds.), Human Rights Regimes in the Americas, Tokyo, United Nations University Press, 2010.

Anaya, Alejandro, El país bajo presión: debatiendo el papel del escrutinio internacional de derechos humanos sobre México, México, CIDE, 2014.

CASLA, Koldo, "El papel de los derechos humanos en la política exterior de y en la Unión Europea: un modelo de análisis", Relaciones Internacionales, núm. 17, 2011.

Chabat, Jorge, "La Iniciativa Mérida y la relación México-Estados. Unidos: en busca de la confianza perdida", en Rafael Velázquez y Juan Pablo Prado (coords.), La Iniciativa Mérida: ¿Nuevo paradigma de cooperación entre México y Estados Unidos en seguridad?, México, UnAM - BUAP, 2009. 
Covarrubias, Ana y Natalia Saltalamacchia, Los derechos humanos y la politica exterior de los paises de América Latina, México, ITAM - Porrúa, 2011.

“Condiciona EU fondos para Iniciativa Mérida”, El Universal, 18 de mayo, 2008.

Negrín, Alejandro, "Una política exterior de derechos humanos para la democracia”, El Cotidiano, núm. 150, 2008.

OJEDA, MARIO, "Vicente Fox: El rompimiento de facto con Cuba", Foro Internacional, vol. 47, núm. 4, 2007.

Pascoe, Ricardo, En el filo. Historia de una crisis diplomática. Cuba 2001-2002, México, Ediciones Sin Nombre, 2004.

Petrich, Blanche, “Orilló Fox a Castro a dejar la cumbre; Bush llegó más tarde”, $L a$ Jornada, 22 de marzo, 2002.

Saltalamacchia, Natalia y Covarrubias, Ana, "La dimensión internacional de la reforma de derechos humanos: antecedentes históricos”, en Miguel Carbonell y Pedro Salazar (coords.), La reforma constitucional de derechos humanos: un nuevo paradigma, México, UnAM, 2011.

VelÁzQUEZ, RAFAEL, "El proyecto de política exterior de Vicente Fox: ¿Continuidad o cambio?”, en Rafael Velázquez (coord.), La politica exterior de México bajo un régimen democrático ¿Cambio o continuidad?, México, Plaza y Valdés, 2002. 